\title{
Generalizations of fractional $q$-Leibniz formulae and applications
}

Zeinab SI Mansour*

\section{"Correspondence:}

zsmansour@ksu.edu.sa

Department of Mathematics,

Faculty of Science, King Saud

University, Riyadh, Saudi Arabia

\begin{abstract}
In this paper we generalize the fractional $q$-Leibniz formula introduced by Agarwal in (Ganita 27(1-2):25-32, 1976) for the Riemann-Liouville fractional q-derivative. This extension is a q-version of a fractional Leibniz formula introduced by Osler in (SIAM J. Appl. Math. 18(3):658-674, 1970). We also introduce a generalization of the fractional q-Leibniz formula introduced by Purohit for the Weyl fractional q-difference operator in (Kyungpook Math. J. 50(4):473-482, 2010). Applications are included.
\end{abstract}

\section{$1 q$-notions and notations}

Let $q$ be a positive number, $0<q<1$. In the following, we follow the notations and notions of $q$-hypergeometric functions, the $q$-gamma function $\Gamma_{q}(x)$, Jackson $q$-exponential functions $E_{q}(x)$, and the $q$-shifted factorial as in $[1,2]$. By a $q$-geometric set $A$, we mean a set that satisfies if $x \in A$, then $q x \in A$. Let $f$ be a function defined on a $q$-geometric set $A$. The $q$-difference operator is defined by

$$
D_{q} f(x):=\frac{f(x)-f(q x)}{x-q x}, \quad x \neq 0
$$

The $n$th $q$-derivative, $D_{q}^{n} f$, can be represented by its values at the points $\left\{q^{j} x, j=0,1, \ldots, n\right\}$ through the identity

$$
D_{q}^{n} f(x)=(-1)^{n}(1-q)^{-n} x^{-n} q^{-n(n-1) / 2} \sum_{r=0}^{n}(-1)^{r}\left[\begin{array}{l}
n \\
r
\end{array}\right]_{q} q^{r(r-1) / 2} f\left(x q^{n-r}\right)
$$

for every $x$ in $A \backslash\{0\}$. After some straightforward manipulations, formula (2) can be written as

$$
D_{q}^{n} f(x)=(1-q)^{-n} x^{-n} \sum_{r=0}^{n} q^{r} \frac{\left(q^{-n} ; q\right)_{r}}{(q ; q)_{r}} f\left(x q^{r}\right) \quad \text { for } x \in A \backslash\{0\} .
$$

Moreover, formula (2) can be inverted through the relation

$$
f\left(x q^{n}\right)=\sum_{k=0}^{n}(-1)^{k}\left[\begin{array}{l}
n \\
k
\end{array}\right]_{q}(1-q)^{k} x^{k} q^{\left(\frac{k}{2}\right)} D_{q}^{k} f(x)
$$

2013 Mansour: licensee Springer. This is an Open Access article distributed under the terms of the Creative Commons Attribution License (http://creativecommons.org/licenses/by/2.0), which permits unrestricted use, distribution, and reproduction in any medium, provided the original work is properly cited. 
Formulas (2) and (4) are well known and follow easily by induction. Jackson [3] introduced an integral denoted by

$$
\int_{a}^{b} f(x) d_{q} x
$$

as a right inverse of the $q$-derivative. It is defined by

$$
\int_{a}^{b} f(t) d_{q} t:=\int_{0}^{b} f(t) d_{q} t-\int_{0}^{a} f(t) d_{q} t, \quad a, b \in \mathbb{C},
$$

where

$$
\int_{0}^{x} f(t) d_{q} t:=(1-q) \sum_{n=0}^{\infty} x q^{n} f\left(x q^{n}\right), \quad x \in \mathbb{C},
$$

provided that the series at the right-hand side of (6) converges at $x=a$ and $b$. In [4], Hahn defined the $q$-integration for a function $f$ over $[0, \infty)$ and $[x, \infty), x>0$, by

$$
\begin{aligned}
& \int_{0}^{\infty} f(t) d_{q} t=(1-q) \sum_{n=-\infty}^{\infty} q^{n} f\left(q^{n}\right), \\
& \int_{x}^{\infty} f(t) d_{q} t=(1-q) \sum_{n=1}^{\infty} x q^{-n}(1-q) f\left(x q^{-n}\right),
\end{aligned}
$$

respectively, provided that the series converges absolutely. Al-Salam [5] defined a fractional $q$-integral operator $K_{q}^{-\alpha}$ by

$$
\begin{aligned}
& K_{q}^{-\alpha} \phi(x):=\frac{q^{-\frac{1}{2} \alpha(\alpha-1)}}{\Gamma_{q}(\alpha)} \int_{x}^{\infty} t^{\alpha-1}(x / t ; q)_{\alpha-1} \phi\left(t q^{1-\alpha}\right) d_{q} t, \\
& K_{q}^{0} \phi(x):=\phi(x),
\end{aligned}
$$

where $\alpha \neq-1,-2, \ldots$, as a generalization of the $q$-Cauchy formula

$$
\begin{aligned}
K_{q}^{-n} \phi(x) & =\int_{x}^{\infty} \int_{x_{n-1}}^{\infty} \cdots \int_{x_{1}}^{\infty} \phi(t) d_{q} t d_{q} x_{1} \cdots d_{q} x_{n-1} \\
& =\frac{q^{-\frac{1}{2} n(n-1)}}{\Gamma_{q}(n)} \int_{x}^{\infty} t^{n-1}(x / t ; q)_{n-1} \phi\left(t q^{1-n}\right) d_{q} t,
\end{aligned}
$$

which he introduced in [6] for a positive integer $n$. Using (7), we can write (8) explicitly as

$$
K_{q}^{-\alpha} \phi(x)=q^{-\frac{\alpha(\alpha+1)}{2}} x^{\alpha}(1-q)^{\alpha} \sum_{k=0}^{\infty}(-1)^{k} q^{\left(\frac{k}{2}\right)}\left[\begin{array}{c}
-\alpha \\
k
\end{array}\right]_{q} \phi\left(x q^{-\alpha-k}\right),
$$

or in a more simple form

$$
K_{q}^{-\alpha} \phi(x)=q^{-\frac{\alpha(\alpha+1)}{2}} x^{\alpha}(1-q)^{\alpha} \sum_{k=0}^{\infty} q^{-k \alpha} \frac{\left(q^{\alpha} ; q\right)_{k}}{(q ; q)_{k}} \phi\left(x q^{-\alpha-k}\right) .
$$


Using (2), we can prove

$$
K_{q}^{n} \phi(x)=(-1)^{n} D_{q}^{n} \phi(x) \quad(n \in \mathbb{N}) .
$$

This paper is organized as follows. In Section 2, we mention in brief some known fractional and $q$-fractional Leibniz formulae. In Section 3, we generalize the fractional $q$-Leibniz formula of the Riemann-Liouville fractional $q$-derivative introduced by Agarwal in [7]. Finally, in Section 4, we extend the fractional $q$-Leibniz formula introduced by Purohit [8] for the $q$-Weyl derivatives of nonnegative integral orders to any real order.

\section{Fractional and $q$-fractional Leibniz formulas}

The Riemann-Liouville fractional $q$-integral operator is introduced by Al-Salam in [5] and later by Agarwal in [9] and defined by

$$
I_{q}^{\alpha} f(x):=\frac{x^{\alpha-1}}{\Gamma_{q}(\alpha)} \int_{0}^{x}(q t / x ; q)_{\alpha-1} f(t) d_{q} t, \quad \alpha \notin\{-1,-2, \ldots\} .
$$

Using (6), (12) reduces to

$$
I_{q}^{\alpha} f(x)=x^{\alpha}(1-q)^{\alpha} \sum_{n=0}^{\infty} q^{n} \frac{\left(q^{\alpha} ; q\right)_{n}}{(q ; q)_{n}} f\left(x q^{n}\right)
$$

which is valid for all $\alpha$. The Riemann-Liouville fractional $q$-derivative of order $\alpha, \alpha>0$, is defined by

$$
D_{q}^{\alpha}=D_{q}^{k} I_{q}^{k-\alpha}, \quad k=\lceil\alpha\rceil .
$$

For the definition of Caputo fractional $q$-derivatives, see [10]. See also [11] for more applications. Liouville [12] introduced the fractional Leibniz rule

$$
I^{\alpha}\{f(x) g(x)\}=\sum_{k=0}^{\infty}\left(\begin{array}{c}
-\alpha \\
k
\end{array}\right) f^{(k)}(x) I^{\alpha+k} g(x),
$$

where

$$
I^{\alpha}\{f(x)\}=\frac{1}{\Gamma(\alpha)} \int_{0}^{x}(x-t)^{\alpha-1} f(t) d t
$$

is the familiar Riemann-Liouville integral operator. While Liouville used Fourier expansions in obtaining (14), Grünwald [13] and Letnikov [14] obtained (14) by a different technique. Other extensions and proofs are in the work of Watanabe [15], Post [16], Bassam [17], and Gaer-Rubel [18]. In a series of papers [19-23], Osler introduced several generalizations of (14). For example, in [19] Osler introduced the fractional Leibniz formula

$$
D_{g(z)}^{\alpha}\{u(z) v(z)\}=\sum_{-\infty}^{\infty}\left(\begin{array}{c}
\alpha \\
\gamma+k
\end{array}\right) D_{g(z)}^{\alpha-\gamma-k} u(z) D_{g(z)}^{\gamma+k} v(z)
$$

which coincides with (14) when we set $g(z)=z, \gamma=0$ and replace $\alpha$ with $-\alpha$ in (15). For an extensive study of the fractional calculus and its applications in physics and control theory, 
see [24-28]. There are two $q$-analogues of the fractional Leibniz rule (14). Al-Salam and Verma [29] introduced the fractional Leibniz formula

$$
I_{q}^{\alpha}(U V)(z)=\sum_{m=0}^{\infty}\left[\begin{array}{c}
-\alpha \\
m
\end{array}\right]_{q} D_{q}^{m} U\left(z q^{-\alpha-m}\right) I_{q}^{\alpha+m} V(z),
$$

formally. An analytic proof of (16) is introduced in [10] where the following theorem is introduced.

Theorem 2.1 Let $U(z)$ be an entire function with q-exponential growth of order $k, k<$ $\ln q^{-1}$, and a finite type $\delta, \delta \in \mathbb{R}$. Let $V$ be a function that satisfies

$$
\sum_{j=0}^{\infty} q^{j}\left|V\left(z q^{j}\right)\right|<\infty \quad(z \in \mathbb{C})
$$

Then (16) holds for $z \in \mathbb{C} \backslash\{0\}$ and $\alpha \in \mathbb{R}$.

For the definition of the q-exponential growth, see [30]. In [7], Agarwal introduced the following fractional $q$-Leibniz formula.

Theorem 2.2 Let $U$ and $V$ be two analytic functions which have power series representations at $z=0$ with radii of convergence $R_{1}$ and $R_{2}$, respectively, and $R=\min \left\{R_{1}, R_{2}\right\}$. Then

$$
I_{q}^{\alpha}(U V)(z)=\sum_{n=0}^{\infty}\left[\begin{array}{c}
-\alpha \\
n
\end{array}\right]_{q} D_{q}^{n} U(z) I_{q}^{\alpha+n} V\left(z q^{n}\right) \quad(|z|<R),
$$

Proof See [7].

Recently, Purohit [31] used (17) to derive a number of summation formulae for the generalized basic hypergeometric functions. In the following section, we introduce a generalization of Agarwal's fractional $q$-Leibniz formula (17). Let $0<R<\infty$ and $D_{R}:=\{z \in \mathbb{C}$ : $|z|<R\}$. In the following, we say that a function $f \in L_{q}^{1}\left(D_{R}\right)$ if

$$
\sum_{j=0}^{\infty} q^{j}\left|f\left(z q^{j}\right)\right|<\infty \quad \text { for all } z \in D_{R} \backslash\{0\}
$$

In [8], Purohit derived a $q$-extension of the Leibniz rule for $q$-derivative via the Weyl $q$-derivative operator defined in (8). He proved that for a nonnegative integer $\alpha$,

$$
K_{q}^{\alpha}(U V)(z)=\sum_{r=0}^{\alpha} \frac{(-1)^{r} q^{r(r+1) / 2}\left(q^{-\alpha} ; q\right)_{r}}{(q ; q)_{r}} K_{q}^{\alpha-r} U(z) K_{q, z}^{r} V\left(z q^{r-\alpha}\right),
$$

where $U(z)=z^{-p_{1}} u(z), V(z)=z^{-p_{2}} v(z), u$ and $v$ are analytic functions having a power series expansion at $z=0$ with radii of convergence $\rho, \rho>0$, and $p_{1}, p_{2} \geq 0$. Purohit established some summation formulae as an application of the fractional Leibniz formula (18) which can be represented as

$$
K_{q}^{\alpha}(U V)(z)=\sum_{r=0}^{\alpha} \frac{\left(q^{-\alpha} ; q\right)_{r}}{(q ; q)_{r}} K_{q}^{\alpha-r} U(z) D_{q^{-1}, z}^{r}\left\{V\left(z q^{\alpha}\right)\right\},
$$


where we used

$$
D_{q^{-1}, z}^{r} V\left(z q^{\alpha}\right)=(-1)^{r} q^{r(r+1) / 2} K_{q, z}^{r} V\left(z q^{r-\alpha}\right)
$$

\section{A generalization for Agarwal's fractional $q$-Leibniz formula}

In this section we introduce a $q$-analogue of the fractional Leibniz formula (15) when $g(z)=z$. The case $\gamma=0$ of the derived fractional $q$-Leibniz formula is the fractional $q$-Leibniz formula (17) introduced by Agarwal [7].

Theorem 3.1 Let $G$ be a branch domain of the logarithmic function. Let $a, b$ be complex numbers and $R$ be a positive number. Let $u$ and $v$ be analytic functions in the disk $D_{R}$. Let $U$ and $V$ be defined in $G \cap D_{R}$ through the relations

$$
U(z)=z^{a} u(z), \quad V(z)=z^{b} v(z) .
$$

If $V(\cdot)$ and $U V(\cdot)$ are in $L_{q}^{1}\left(D_{R}\right)$, then

$$
\begin{aligned}
& I_{q}^{\alpha} U V(z) \\
& \quad=\left.z^{\gamma} \frac{\Gamma_{q}(\alpha)}{\Gamma_{q}(\alpha-\gamma)} \sum_{m=0}^{\infty}\left[\begin{array}{c}
-\alpha+\gamma \\
m
\end{array}\right]_{q} D_{q}^{m}\left(\left(q^{\alpha-\gamma} \xi / z ; q\right)_{\gamma} U(\xi)\right)\right|_{\xi=z}\left(I_{q}^{\alpha-\gamma+m}\right) V\left(z q^{m}\right),
\end{aligned}
$$

where $z \in G \cap D_{R}$, and $\alpha, \gamma \in \mathbb{R}$.

Remark 3.2 It is worthwhile to notice that if we set $\gamma=0$ in (21), we obtain Agarwal's fractional Leibniz rule (17) with less restrictive conditions on the functions $U(z)$ and $V(z)$. Actually, the special case $\gamma=0$ of Theorem 3.1 is an extension of the result given by Manocha and Sharma in [32].

Proof Since $V, U V$ are in $L_{q}^{1}\left(D_{R}\right)$, then

$$
z^{a} V \in L_{q}^{1}\left(D_{R}\right), \quad \operatorname{Re}(b)>-1 \quad \text { and } \quad \operatorname{Re}(a+b)>-1 .
$$

From (13) we obtain

$$
I_{q}^{\alpha}(U V)(z)=z^{\alpha}(1-q)^{\alpha} \sum_{n=0}^{\infty} q^{n} \frac{\left(q^{\alpha} ; q\right)_{n}}{(q ; q)_{n}} U\left(z q^{n}\right) V\left(z q^{n}\right)
$$

Substituting with

$$
\frac{\left(q^{\alpha} ; q\right)_{n}}{(q ; q)_{n}}=\frac{\left(q^{\alpha-\gamma} ; q\right)_{n}}{(q ; q)_{n}}\left(q^{\alpha} ; q\right)_{-\gamma}\left(q^{\alpha-\gamma+n} ; q\right)_{\gamma}
$$

into (22), we obtain

$$
I_{q}^{\alpha}(U V)(z)=z^{\alpha}(1-q)^{\alpha}\left(q^{\alpha} ; q\right)_{-\gamma} \sum_{n=0}^{\infty} q^{n} \frac{\left(q^{\alpha-\gamma} ; q\right)_{n}}{(q ; q)_{n}}\left(q^{\alpha-\gamma+n} ; q\right)_{\gamma} U\left(z q^{n}\right) V\left(z q^{n}\right) .
$$


The existence of $U V$ in the space $L_{q}^{1}\left(D_{R}\right)$ guarantees that the series in (22) or in (23) converges absolutely for all $z \in D_{R} \backslash\{0\}$. Replace $x$ in (4) with $\xi$ and then let

$$
f(\xi)=\left(q^{\alpha-\gamma} \xi / z ; q\right)_{\gamma} U(\xi)
$$

Consequently,

$$
\begin{aligned}
& \left(q^{\alpha-\gamma+n} ; q\right)_{\gamma} U\left(z q^{n}\right) \\
& \quad=\left.\sum_{k=0}^{n}(-1)^{k}(1-q)^{k}\left[\begin{array}{l}
n \\
k
\end{array}\right]_{q} q^{k(k-1) / 2} z^{k} D_{q}^{k}\left(\left(q^{\alpha-\gamma} \xi / z ; q\right)_{\gamma} U(\xi)\right)\right|_{\xi=z^{*}}
\end{aligned}
$$

Then substituting (24) into (23), we get

$$
\begin{aligned}
I_{q}^{\alpha}(U V)(z)= & z^{\alpha}(1-q)^{\alpha}\left(q^{\alpha} ; q\right)_{-\gamma} \sum_{n=0}^{\infty} q^{n} \frac{\left(q^{\alpha-\gamma} ; q\right)_{n}}{(q ; q)_{n}} V\left(z q^{n}\right) \\
& \times\left.\sum_{k=0}^{n}(-1)^{k}(1-q)^{k}\left[\begin{array}{l}
n \\
k
\end{array}\right]_{q} q^{k(k-1) / 2} z^{k} D_{q}^{k}\left(\left(q^{\alpha-\gamma} \xi / z ; q\right)_{\gamma} U(\xi)\right)\right|_{\xi=z^{*}}
\end{aligned}
$$

Using (2), we obtain

$$
\begin{aligned}
\left.D_{q}^{k}\left(\left(q^{\alpha-\gamma} \frac{\xi}{z} ; q\right)_{\gamma} U(\xi)\right)\right|_{\xi=k}= & (-1)^{k}(1-q)^{-k} z^{a-k} q^{-\frac{k(k-1)}{2}}\left(q^{\alpha-\gamma} ; q\right)_{\gamma} \\
& \times \sum_{r=0}^{k} q^{\frac{r(r-1)}{2}}\left[\begin{array}{l}
k \\
r
\end{array}\right]_{q} \frac{\left(q^{\alpha} ; q\right)_{k-r}}{\left(q^{\alpha-\gamma} ; q\right)_{k-r}} q^{(k-r) a} u\left(z q^{k-r}\right) .
\end{aligned}
$$

Therefore, since $u(z)$ is analytic in $D_{R}$, there exists $M>0$ such that

$$
\begin{aligned}
& \left|D_{q}^{k}\left(\left(q^{\alpha-\gamma} \frac{\xi}{z} ; q\right)_{\gamma} U(\xi)\right)\right|_{\xi=k} \mid \\
& \quad \leq(1-q)^{-k}|z|^{\operatorname{Re}(a)-k} q^{k \operatorname{Re} a-\frac{k(k-1)}{2}} M \sum_{r=0}^{k} q^{\frac{r(r-1)}{2}}\left[\begin{array}{c}
k \\
r
\end{array}\right]_{q} q^{-r \operatorname{Re} a} \\
& \quad=M(1-q)^{-k}|z|^{\operatorname{Re}(a)-k} q^{k \operatorname{Re} a-\frac{k(k-1)}{2}}\left(-q^{-\operatorname{Re}(a)} ; q\right)_{k} \\
& \quad \leq M(1-q)^{-k}|z|^{\operatorname{Re}(a)-k} q^{k \operatorname{Re} a-\frac{k(k-1)}{2}}\left(-q^{-\operatorname{Re}(a)} ; q\right)_{\infty} .
\end{aligned}
$$

Consequently,

$$
\begin{aligned}
& \sum_{k=0}^{n}(1-q)^{k}\left[\begin{array}{l}
n \\
k
\end{array}\right]_{q} q^{k(k-1) / 2}\left|z^{k} D_{q}^{k}\left(\left(q^{\alpha-\gamma} \frac{\xi}{z} ; q\right)_{\gamma} U(\xi)\right)\right|_{\xi=z} \mid \\
& \quad \leq M|z|^{\operatorname{Re}(a)} \frac{\left(-q^{\operatorname{Re}(a)} ; q\right)_{\infty}}{(q ; q)_{\infty}^{2}} \frac{1-q^{(n+1) \operatorname{Re}(a)}}{1-q^{\operatorname{Re}(a)}} .
\end{aligned}
$$


Set $F(\xi):=\left(q^{\alpha-\gamma} \frac{\xi}{z} ; q\right)_{\gamma} U(\xi)$. Then substituting (27) into (25), we obtain

$$
\begin{aligned}
& \sum_{n=0}^{\infty} \frac{q^{n}\left|\left(q^{\alpha-\gamma} ; q\right)_{n}\right|}{(q ; q)_{n}}\left|V\left(z q^{n}\right)\right| \sum_{k=0}^{n}(1-q)^{k}\left[\begin{array}{c}
n \\
k
\end{array}\right]_{q} q^{k(k-1) / 2}\left|z^{k} D_{q}^{k} F(z)\right| \\
& \quad \leq M|z|^{\operatorname{Re}(a)} \frac{\left(-q^{\alpha-\gamma} ; q\right)_{\infty}\left(-q^{-\operatorname{Re}(a)} ; q\right)_{\infty}}{(q ; q)_{\infty}^{3}} \sum_{n=0}^{\infty} \frac{1-q^{(n+1) \operatorname{Re}(a)}}{1-q^{\operatorname{Re}(a)}} q^{n}\left|V\left(z q^{n}\right)\right| .
\end{aligned}
$$

The last series converges for all $z \in D_{R} \backslash\{0\}$ since $V, z^{a} V \in L_{q}^{1}\left(D_{R}\right)$. Consequently, the series in (28) is absolutely convergent, and we can interchange the order of summations in (25). This leads to

$$
\begin{aligned}
& I_{q}^{\alpha}(U V)(z) \\
& =z^{\alpha}(1-q)^{\alpha}\left(q^{\alpha} ; q\right)_{1-\gamma} \sum_{k=0}^{\infty}(-1)^{k} q^{k(k-1) / 2} z^{k} \frac{(1-q)^{k}}{(q ; q)_{k}} D_{q}^{k} F(z) \sum_{n=k}^{\infty} q^{n} \frac{\left(q^{\alpha-\gamma} ; q\right)_{n}}{(q ; q)_{n-k}} V\left(z q^{n}\right) \\
& =z^{\alpha}(1-q)^{\alpha}\left(q^{\alpha} ; q\right)_{-\gamma} \sum_{k=0}^{\infty}(-1)^{k} q^{k(k+1) / 2} z^{k} \frac{(1-q)^{k}}{(q ; q)_{k}} \\
& \quad \times D_{q}^{k} F(z) \sum_{j=0}^{\infty} q^{j} \frac{\left(q^{\alpha-\gamma} ; q\right)_{j+k}}{(q ; q)_{j}} V\left(z q^{j+k}\right) .
\end{aligned}
$$

Since

$$
\left(I_{q}^{\alpha-\gamma+k} V\right)\left(z q^{k}\right)=\left(z q^{k}\right)^{\alpha-\gamma+k}(1-q)^{\alpha-\gamma+k} \sum_{j=0}^{\infty} q^{j} \frac{\left(q^{\alpha-\gamma+k} ; q\right)_{j}}{(q ; q)_{j}} V\left(z q^{j+k}\right)
$$

and

$$
\left(q^{\alpha-\gamma} ; q\right)_{j+k}=\left(q^{\alpha-\gamma} ; q\right)_{k}\left(q^{\alpha-\gamma+k} ; q\right)_{j}
$$

the substitution with the last two identities in (29) gives

$$
\begin{aligned}
& I_{q}^{\alpha}(U V)(z) \\
& =z^{\gamma}(1-q)^{\gamma}\left(q^{\alpha} ; q\right)_{-\gamma} \sum_{k=0}^{\infty}(-1)^{k} q^{-k(k-1) / 2+k(-\alpha+\gamma)} \frac{\left(q^{\alpha-\gamma} ; q\right)_{k}}{(q ; q)_{k}} \\
& \left.\quad \times\left(I_{q}^{\alpha-\gamma+k} V\right)\left(z q^{k}\right) D_{q}^{k}\left(\left(q^{\alpha-\gamma} \frac{\xi}{z} ; q\right)\right)_{\gamma} U(\xi)\right)\left.\right|_{\xi=z} \\
& \left.=z^{\gamma}(1-q)^{\gamma}\left(q^{\alpha} ; q\right)_{-\gamma} \sum_{k=0}^{\infty}\left[\begin{array}{c}
-\alpha+\gamma \\
k
\end{array}\right]_{q}\left(I_{q}^{\alpha-\gamma+k} V\right)\left(z q^{k}\right) D_{q}^{k}\left(\left(q^{\alpha-\gamma} \frac{\xi}{z} ; q\right)\right)_{\gamma} U(\xi)\right)\left.\right|_{\xi=z},
\end{aligned}
$$

and the theorem follows.

Example 3.3 Let $\gamma, \lambda$, $\mu$, and $\alpha$ be complex numbers satisfying

$$
\operatorname{Re}(\lambda)>0, \quad \operatorname{Re}(\lambda+\mu)>0, \quad \mu \notin \mathbb{N}_{0} \quad \text { and } \quad \operatorname{Re}(\alpha)>0 .
$$


Then

$$
\begin{aligned}
& \frac{\Gamma_{q}(\lambda+\mu) \Gamma_{q}(\alpha) \Gamma_{q}(\lambda+\alpha-\gamma)}{\Gamma_{q}(\alpha-\gamma) \Gamma_{q}(\lambda) \Gamma_{q}(\lambda+\mu+\alpha)} \\
& \quad=\sum_{m=0}^{\infty} q^{m(\lambda+\mu)} \frac{\left(q^{\alpha-\gamma}, q^{-\mu} ; q\right)_{m}}{\left(q, q^{\lambda+\alpha-\gamma} ; q\right)_{m}}{ }_{2} \phi_{1}\left(q^{-\gamma}, q^{\mu+1} ; q^{\mu-m+1} ; q, q^{\alpha}\right) .
\end{aligned}
$$

Proof We prove the identity by using Theorem 3.1. Take $U(z)=z^{\mu}$ and $v(z)=z^{\lambda-1}$. Then

$$
\begin{aligned}
& D_{q}^{m}\left\{\left(q^{\alpha-\gamma} \frac{\xi}{z} ; q\right)_{\gamma} \xi^{\mu}\right\} \\
& =D_{q}^{m} \sum_{k=0}^{\infty} \frac{\left(q^{-\gamma} ; q\right)_{k}}{(q ; q)_{k}}\left(\frac{q^{\alpha}}{z}\right)^{k} \xi^{\mu+k} \\
& =\sum_{k=0}^{\infty} \frac{\left(q^{-\gamma} ; q\right)_{k}}{(q ; q)_{k}}\left(\frac{q^{\alpha}}{z}\right)^{k} \frac{\Gamma_{q}(\mu+k+1)}{\Gamma_{q}(\mu+k-m+1)} \xi^{\mu+k-m} .
\end{aligned}
$$

Hence,

$$
\begin{aligned}
& \left.D_{q}^{m}\left\{\left(q^{\alpha-\gamma} \frac{\xi}{z} ; q\right)_{\gamma} \xi^{\mu}\right\}\right|_{\xi=z} \\
& \quad=z^{\mu-m} \frac{\Gamma_{q}(\mu+1)}{\Gamma_{q}(\mu-m+1)}{ }_{2} \phi_{1}\left(q^{-\gamma}, q^{\mu+1} ; q^{\mu-m+1} ; q, q^{\alpha}\right) \\
& =(-1)^{m} \frac{\left(q^{-\mu} ; q\right)_{m}}{(1-q)^{m}} q^{m \mu-\left(\begin{array}{c}
m \\
2
\end{array}\right)} z^{\mu-m}{ }_{2} \phi_{1}\left(q^{-\gamma}, q^{\mu+1} ; q^{\mu-m+1} ; q, q^{\alpha}\right)
\end{aligned}
$$

and

$$
\begin{aligned}
\left(I_{q}^{\alpha-\gamma+m} V\right)\left(z q^{m}\right) & =\frac{\Gamma_{q}(\lambda)}{\Gamma_{q}(\lambda+\alpha-\gamma+m)}\left(z q^{m}\right)^{\lambda+\alpha-\gamma+m-1} \\
& =\frac{\Gamma_{q}(\lambda)}{\Gamma_{q}(\lambda+\alpha-\gamma)} \frac{(1-q)^{m}}{\left(q^{\lambda+\alpha-\gamma} ; q\right)_{m}}\left(z q^{m}\right)^{\lambda+\alpha-\gamma+m-1}
\end{aligned}
$$

Then applying Theorem 3.1 gives

$$
\begin{aligned}
I_{q}^{\alpha}(U V)(z)= & z^{\mu+\lambda+\alpha-1} \frac{\Gamma_{q}(\alpha) \Gamma_{q}(\lambda)}{\Gamma_{q}(\alpha-\gamma) \Gamma_{q}(\lambda+\alpha-\gamma)} \\
& \times \sum_{m=0}^{\infty} q^{m(\lambda+\mu)} \frac{\left(q^{\alpha-\gamma}, q^{-\mu} ; q\right)_{m}}{\left(q, q^{\lambda+\alpha-\gamma} ; q\right)_{m}}{ }_{2} \phi_{1}\left(q^{-\gamma}, q^{\mu+1} ; q^{\mu-m+1} ; q, q^{\alpha}\right) .
\end{aligned}
$$

On the other hand,

$$
I_{q}^{\alpha} z^{\lambda+\mu-1}=\frac{\Gamma_{q}(\lambda+\mu)}{\Gamma_{q}(\lambda+\mu+\alpha)} z^{\lambda+\mu+\alpha-1}
$$

Equating (33) and (34) gives (30). 
Example 3.4 For complex numbers $a, b, A, B, d$, and $D$ such that $\operatorname{Re}(b)>-1, \operatorname{Re}(B)>0$, and $\operatorname{Re}(b+B)>1$,

$$
\begin{aligned}
& { }_{2} \phi_{1}\left(q^{a+A}, q^{b+B} ; q^{d+D} ; q, q^{-(a+A)} z\right) \\
& =(z ; q)_{-a} \frac{\Gamma_{q}(d+D) \Gamma_{q}(B)}{\Gamma_{q}(b+B) \Gamma_{q}(d+B-b)}(1-q)^{B-D} \\
& \quad \times \sum_{m=0}^{\infty}(-1)^{m} q^{\left(\begin{array}{c}
m \\
2
\end{array}\right)} q^{m B} \frac{\left(q^{b-d} ; q\right)_{m}}{(q ; q)_{m}\left(q^{d+D-b} ; q\right)_{m}} \\
& \quad \times{ }_{3} \phi_{2}\left(q^{-m}, q^{d+D-b-B}, z q^{-a} ; q^{d-b}, z ; q, q^{b+1}\right)_{2} \phi_{1}\left(q^{A}, q^{B} ; q^{d+B-b+m} ; q, q^{-a-A+m}\right),
\end{aligned}
$$

for $\left|z q^{-a-A}\right|<1$.

Proof The previous identity follows by taking

$$
U(z)=z^{b}(z ; q)_{-a}, \quad V(z)=z^{B-1}\left(z q^{-a} ; q\right)_{-A},
$$

and applying Theorem 3.1 with

$$
\alpha=d+D-b-B, \quad \gamma=D-B
$$

Then using (3), we obtain

$$
\begin{aligned}
\left.D_{q}^{m}\left(\left(q^{d-b} \frac{\xi}{z} ; q\right)\right)_{D-B} \xi^{b}(\xi ; q)_{-a}\right)\left.\right|_{\xi=z}= & (1-q)^{-m} z^{-m+b}(z ; q)_{-a} \frac{\left(q^{d-b} ; q\right)_{\infty}}{\left(q^{d-b+D-B} ; q\right)_{\infty}} \\
& \times{ }_{3} \phi_{2}\left(q^{-m}, q^{d-b+D-B}, z q^{-a} ; q^{d-b}, z ; q, q^{b+1}\right) .
\end{aligned}
$$

In addition,

$$
\begin{aligned}
\left(I_{q}^{\alpha-\gamma+m} V\right)\left(z q^{m}\right)= & z^{d-b+\beta-1+m} q^{m^{2}+(d-b+\beta-1) m} \frac{\Gamma_{q}(\beta)}{\Gamma_{q}(B+m+d-b)} \\
& \times{ }_{2} \phi_{1}\left(q^{A}, q^{\beta} ; q^{m-b} ; q, q^{m-a-A}\right)
\end{aligned}
$$

and

$$
\left[\begin{array}{c}
\gamma-\alpha \\
m
\end{array}\right]_{q}=(-1)^{m} q^{(\gamma-\alpha) m} q^{\left(\begin{array}{c}
-m \\
2
\end{array}\right)} \frac{\left(q^{\alpha-\gamma} ; q\right)_{m}}{(q ; q)_{m}} .
$$

Substituting with (36)-(38) into (21), we obtain

$$
\begin{aligned}
I_{q}^{\alpha} U V(z)= & z^{d+D-1}(1-q)^{D-B}(z ; q)_{-a} \frac{\Gamma_{q}(\beta)}{\Gamma_{q}(B+d-b)} \\
& \times \sum_{m=0}^{\infty}(-1)^{m} q^{\left(\begin{array}{c}
m \\
2
\end{array}\right)} q^{m B} \frac{\left(q^{b-d} ; q\right)_{m}}{(q ; q)_{m}\left(q^{d+D-b} ; q\right)_{m}} \\
& \times{ }_{3} \phi_{2}\left(q^{-m}, q^{d+D-b-B}, z q^{-a} ; q^{d-b}, z ; q, q^{b+1}\right) \\
& \times{ }_{2} \phi_{1}\left(q^{A}, q^{B} ; q^{d+B-b+m} ; q, q^{-a-A+m}\right) .
\end{aligned}
$$


On the other hand,

$$
I_{q}^{\alpha} U V(z)=\frac{\Gamma_{q}(b+B)}{\Gamma_{q}(d+D)} z_{2}^{d+D-1} \phi_{1}\left(q^{a+A}, q^{b+B} ; q^{d+D} ; q, q^{-(a+A)}\right) .
$$

Combining (39) and (40), we obtain (35).

\section{A q-extension of the Leibniz rule via Weyl-type of a $q$-derivative operator}

In this section, we prove that the $q$-expansion in (18) can be derived for any $\alpha \in \mathbb{R}$. The proof we introduce is completely different from the one introduced by Purohit for nonnegative integer values of $\alpha$. We start with characterizing a sufficient class of functions for which $K_{q}^{-\alpha}$ exists for some $\alpha$.

Definition 4.1 Let $\alpha \in \mathbb{C}$ and let $f$ be a function defined on a $q^{-1}$-geometric set $A$. We say that $f$ is of class $S_{q, \alpha}$ if there exists $\mu \in \mathbb{C}, \operatorname{Re} \mu>\operatorname{Re} \alpha$ such that

$$
f\left(x q^{-n}\right)=O\left(q^{n \mu}\right) \quad \text { as } n \rightarrow \infty, x \in A .
$$

Proposition 4.2 If $\alpha \in \mathbb{Z}$, then $K_{q}^{-\alpha} f$ exists for any function $f$ defined on $(0, \infty)$. If $\alpha \notin \mathbb{Z}$ and $f \in S_{q, \alpha}$, then $K_{q}^{-\alpha} f$ exists.

Proof If $\alpha \in \mathbb{Z}$, then by (11), $K_{q}^{-\alpha} f$ exists for any functions $f$ defined on a $(0, \infty)$. If $\alpha \notin \mathbb{Z}$ and $f \in S_{q, \alpha}$, then for each $x>0$, there exists a constant $C>0, C$ depends on $x$ and $\alpha$, such that

$$
\left|f\left(x q^{-n-\alpha}\right)\right| \leq C q^{n \mu}
$$

Applying the previous inequality in (10) gives

$$
\begin{aligned}
\left|K_{q}^{-\alpha} f(x)\right| & \leq C q^{-\alpha(\alpha+1) / 2}|x|^{\alpha}(1-q)^{\alpha} \frac{\left(-q^{\operatorname{Re} \alpha} ; q\right)_{\infty}}{(q ; q)_{\infty}} \sum_{k=0}^{\infty} q^{k(\operatorname{Re} \mu-\operatorname{Re} \alpha)} \\
& \leq C q^{-\alpha(\alpha+1) / 2}|x|^{\alpha}(1-q)^{\alpha} \frac{\left(-q^{\operatorname{Re} \alpha} ; q\right)_{\infty}}{\left(1-q^{(\operatorname{Re} \mu-\operatorname{Re} \alpha)}\right)(q ; q)_{\infty}}
\end{aligned}
$$

In the following, we define a sufficient class of functions $\mathfrak{S}_{q, \mu}$ for which $K_{q}^{-\alpha} f$ exists for all $\alpha$.

Definition 4.3 Let $f$ be a function defined on a $q^{-1}$-geometric set $A$. We say that $f$ is in the class $\mathfrak{S}_{q, \mu}$ if there exist $\mu>0$ and $\nu \in \mathbb{R}$ such that for each $x \in A$,

$$
\left|f\left(x q^{-n}\right)\right|=O\left(q^{\mu n(n+v)}\right) \quad \text { as } n \rightarrow \infty
$$

It is clear that if $f \in \mathfrak{S}_{q, \mu}$, then $f \in S_{q, \alpha}$ for all $\alpha$. The spaces $S_{q, \alpha}$ and $\mathfrak{S}_{q, \mu}$ are $q$-analogues of the spaces of fairly good functions and good functions, respectively, introduced by Lighthill [33, p.15], see also [34, Chapter VII]. 
Example 4.4 An example of a function in a class $S_{q, 1 / 2}$ is any function of the form

$$
\frac{P_{n}(x)}{(a x ; q)_{\infty}} \text { for all } n \in \mathbb{N}_{0}
$$

where $P_{n}(x)$ is a polynomial of degree $n$ and $a$ is a constant such that $a x q^{k} \neq 1$ for all $k \in \mathbb{N}_{0}$.

The keynotes in proving the generalization of Purohit $q$-fractional Leibniz formula are two identities. The first one is

$$
K_{q}^{\alpha} z^{-p}=q^{\alpha(1-\alpha) / 2} q^{-\alpha p} z^{-\alpha-p} \frac{\Gamma_{q}(\alpha+p)}{\Gamma_{q}(p)}
$$

which holds for any $p \in \mathbb{R}$ when $\alpha \in \mathbb{N}$ or holds when $\alpha+p>0$. The proof of (41) follows from (10) by replacing $\alpha$ with $-\alpha$, x with $z$, and setting $\phi(z)=z^{-p}$. The second identity follows from the formula (4) with $q$ replaced with $q^{-1}$ and $x$ with $z$. That is,

$$
\begin{aligned}
f\left(z q^{-n}\right) & =\sum_{k=0}^{n}\left(q^{-1}-1\right)^{k} q^{-\left(\begin{array}{l}
k \\
2
\end{array}\right)}\left[\begin{array}{l}
n \\
k
\end{array}\right]_{q^{-1}} z^{k} D_{q^{-1}}^{k} f(z) \\
& =\sum_{k=0}^{n} q^{k(k-1) / 2}(1-q)^{k} q^{-n k}\left[\begin{array}{l}
n \\
k
\end{array}\right]_{q} z^{k} D_{q^{-1}}^{k} f(z),
\end{aligned}
$$

where we use [1, Eq. (I.47)]

$$
\left[\begin{array}{l}
n \\
k
\end{array}\right]_{q^{-1}}=\left[\begin{array}{l}
n \\
k
\end{array}\right]_{q} q^{k^{2}-n k}
$$

The identity in (41) leads to the following result.

Lemma 4.5 Let $p$ and $\alpha$ be such that $0<\operatorname{Re} p<1$ and $\alpha>\operatorname{Re} p$. Let $G$ be the principal branch of the logarithmic function and let $D_{R}:=\{z \in \mathbb{C}:|z|>R\} \cap G$. Assume that

$$
U(z)=z^{p} \sum_{j=0}^{\infty} a_{j} z^{-j}
$$

is analytic on $D_{R}$. Let

$$
\Omega_{\alpha}=\left\{z \in D_{R}: q^{\alpha} z \in D_{R}\right\}
$$

Then $K_{q}^{\alpha} U(z)$ exists for all $z \in \Omega_{\alpha}$ and is equal to

$$
K_{q}^{\alpha} U(z)=q^{\alpha p} \frac{\Gamma_{q}(\alpha-p)}{\Gamma_{q}(-p)} z^{p-\alpha} \sum_{j=0}^{\infty} a_{j} \frac{\left(q^{-p} ; q\right)_{j}}{\left(q^{\alpha-p} ; q\right)_{j}} q^{-\alpha j} z^{-j}
$$

Proof From (10) we find that

$$
K_{q}^{\alpha} U(z)=q^{\alpha(1-\alpha) / 2} q^{\alpha p} z^{p-\alpha}(1-q)^{-\alpha} \sum_{k=0}^{\infty} q^{k(\alpha-p)} \frac{\left(q^{-\alpha} ; q\right)_{k}}{(q ; q)_{k}} \sum_{j=0}^{\infty} a_{j} q^{(-\alpha+k) j}
$$


From the assumptions of the present lemma, we can easily deduce that the double series in (44) is absolutely convergent for all $z \in \Omega_{\alpha}$. Hence, we can interchange the order of summations in (44). This and the $q$-binomials theorem [1, Eq. (1.3.2)] give

$$
\begin{aligned}
K_{q}^{\alpha} U(z) & =q^{\alpha(1-\alpha) / 2} q^{\alpha p} z^{p-\alpha}(1-q)^{-\alpha} \sum_{j=0}^{\infty} a_{j} q^{-\alpha j} z^{-j} \sum_{k=0}^{\infty} q^{k \alpha-k p+k j} \frac{\left(q^{-\alpha} ; q\right)_{j}}{(q ; q)_{j}} \\
& =q^{\alpha(1-\alpha) / 2} q^{\alpha p} z^{p-\alpha}(1-q)^{-\alpha} \sum_{j=0}^{\infty} a_{j} q^{-\alpha j} z^{-j} \frac{\left(q^{-p+j} ; q\right)_{\infty}}{\left(q^{\alpha-p+j} ; q\right)_{\infty}}
\end{aligned}
$$

Simple manipulations give (43).

Lemma 4.6 Let $p, \alpha, G, U, D_{R}$, and $\Omega_{\alpha}$ be as in Lemma 4.5. Then

$$
\sum_{j=0}^{\infty} q^{\alpha j}\left|U\left(z q^{\alpha-j}\right)\right|<\infty \quad \forall z \in \Omega_{\alpha}
$$

Proof The proof is easy and is omitted.

Theorem 4.7 Let $U$ and $V$ be functions defined on $a q^{-1}$-geometric set $A$ and let $\alpha \in \mathbb{R}$. Assume that $U V \in S_{q, \alpha}$ and $U \in S_{q, \mu}, \mu>\frac{1}{2}$. Then

$$
K_{q}^{\alpha} U V(z)=\sum_{m=0}^{\infty} \frac{\left(q^{-\alpha} ; q\right)_{m}}{(q ; q)_{m}} K_{q}^{\alpha-m} U(z) D_{q^{-1}, z}^{m}\left\{V\left(z q^{\alpha}\right)\right\}
$$

for all $z \in A$ and for all $\alpha \in \mathbb{R}$. If $\mu=1 / 2$, then (45) may not hold for all $\alpha$ on $\mathbb{R}$ but only for $\alpha$ in a subdomain of $\mathbb{R}$.

Proof Let $z \in q^{-\alpha} A$ be arbitrary but fixed. Since $U V \in S_{q, \alpha}$, then

$$
\sum_{k=0}^{\infty} q^{k \alpha}\left|U\left(z q^{\alpha-k}\right) V\left(z q^{\alpha-k}\right)\right|<\infty
$$

From (10),

$$
\begin{aligned}
& \left(K_{q}^{\alpha} U V\right)(z) \\
& \quad=q^{\alpha(1-\alpha) / 2}(1-q)^{-\alpha} z^{-\alpha} \sum_{m=0}^{\infty} q^{\alpha m} \frac{\left(q^{-\alpha} ; q\right)_{m}}{(q ; q)_{m}} U\left(z q^{\alpha-m}\right) V\left(z q^{\alpha-m}\right) .
\end{aligned}
$$

Applying (42) with $f(z)=V\left(z q^{\alpha}\right)$ yields

$$
\begin{aligned}
\left(K_{q}^{\alpha} U V\right)(z)= & q^{\alpha(1-\alpha) / 2}(1-q)^{-\alpha} z^{-\alpha} \sum_{m=0}^{\infty} q^{\alpha m} \frac{\left(q^{-\alpha} ; q\right)_{m}}{(q ; q)_{m}} U\left(z q^{\alpha-m}\right) \\
& \times \sum_{j=0}^{m} q^{j(1-j) / 2}(1-q)^{j} q^{-m j}\left[\begin{array}{c}
m \\
j
\end{array}\right]_{q} z^{j} D_{q^{-1}, z}^{j}\left\{V\left(z q^{\alpha}\right)\right\} .
\end{aligned}
$$


From the assumptions on the function $U$, there exists a constant $C_{1}>0$ and $v \in \mathbb{R}$ such that

$$
\left|U\left(z q^{\alpha-m}\right)\right| \leq C_{1} q^{\mu m(m+v)} .
$$

Using (2) with $\left(q^{-1}\right.$ instead of $\left.q\right)$, we obtain

$$
z^{j} D_{q^{-1, z}}^{j}\left\{V\left(z q^{\alpha}\right)\right\} \leq C_{2}
$$

Consequently, the double series on (47) is bounded from above by

$$
\begin{aligned}
& C_{1} C_{2} \frac{\left(-q^{-\operatorname{Re} \alpha} ; q\right)_{\infty}}{(q ; q)_{\infty}} \sum_{m=0}^{\infty} q^{\operatorname{Re} \alpha m} q^{\mu m(m+v)} \sum_{j=0}^{m} q^{j(1-j) / 2}\left[\begin{array}{c}
m \\
j
\end{array}\right]_{q} q^{-m j} \\
& \leq C_{1} C_{2} \frac{\left(-q^{-\operatorname{Re} \alpha} ; q\right)_{\infty}}{(q ; q)_{\infty}} \sum_{m=0}^{\infty} q^{\operatorname{Re} \alpha m} q^{\mu m(m+v)}\left(-q^{-m} ; q\right)_{m} \\
& =C_{1} C_{2} \frac{\left(-q^{-\operatorname{Re} \alpha} ; q\right)_{\infty}(-q ; q)_{\infty}}{(q ; q)_{\infty}} \sum_{m=0}^{\infty} q^{\operatorname{Re} \alpha m} q^{\mu m(m+v)} q^{-m(m+1) / 2},
\end{aligned}
$$

where we applied the identity $c f .$, e.g., $[1, \mathrm{p} .11]$,

$$
(a ; q)_{n}:=\sum_{k=0}^{n}(-1)^{k}\left[\begin{array}{l}
n \\
k
\end{array}\right]_{q} q^{\frac{k(k-1)}{2}} a^{k}
$$

Now, it is clear that if $\mu>1 / 2$, then the series on the most right-hand side of (48) is convergent for all $\alpha \in \mathbb{C}$. On the other hand, it is convergent only for $\operatorname{Re} \alpha>-v+\frac{1}{2}$ when $\mu=\frac{1}{2}$. Therefore, we can interchange the order of summation in the series on the right-hand side of (47). This gives

$$
\begin{aligned}
\left(K_{q}^{\alpha} U V\right)(z)= & q^{\alpha(1-\alpha) / 2}(1-q)^{-\alpha} z^{-\alpha} \\
& \times \sum_{j=0}^{\infty} \frac{\left(q^{-\alpha} ; q\right)_{j}}{(q ; q)_{j}} z^{j}(1-q)^{j} D_{q^{-1}, z}^{j} V\left(z q^{\alpha}\right) \\
& \times \sum_{r=0}^{\infty} q^{(\alpha-j) r} \frac{\left(q^{-\alpha+j} ; q\right)_{r}}{(q ; q)_{r}} U\left(z q^{\alpha-j-r}\right) .
\end{aligned}
$$

But

$$
\sum_{r=0}^{\infty} q^{(\alpha-j) r} \frac{\left(q^{-\alpha+j} ; q\right)_{r}}{(q ; q)_{r}} U\left(z q^{\alpha-j-r}\right)=z^{\alpha-j}(1-q)^{\alpha-j} q^{(\alpha-j)(\alpha-j-1) / 2} K_{q}^{\alpha-j} V(z)
$$

Combining this latter identity with (50) yields the theorem.

Example 4.8 Let $\gamma, \lambda, \mu$, and $\alpha$ be complex numbers satisfying

$$
\operatorname{Re}(\lambda)>0, \quad \operatorname{Re}(\lambda+\mu)>0, \quad \mu \notin \mathbb{N}_{0} \quad \text { and } \quad \operatorname{Re}(\alpha)>0 .
$$


Then

$$
\begin{aligned}
& \frac{\Gamma_{q}(\lambda+\mu) \Gamma_{q}(\alpha) \Gamma_{q}(\lambda+\alpha-\gamma)}{\Gamma_{q}(\alpha-\gamma) \Gamma_{q}(\lambda) \Gamma_{q}(\lambda+\mu+\alpha)} \\
& \quad=\sum_{m=0}^{\infty} q^{m(\lambda+\mu)} \frac{\left(q^{\alpha-\gamma}, q^{-\mu} ; q\right)_{m}}{\left(q, q^{\lambda+\alpha-\gamma} ; q\right)_{m}}{ }_{2} \phi_{1}\left(q^{-\gamma}, q^{\mu+1} ; q^{\mu-m+1} ; q, q^{\alpha}\right) .
\end{aligned}
$$

Proof We prove the identity by using Theorem 3.1. Take $U(z)=z^{\mu}$ and $v(z)=z^{\lambda-1}$. Then

$$
\begin{aligned}
& D_{q}^{m}\left(q^{\alpha-\gamma} \frac{\xi}{z} ; q\right)_{\gamma} \xi^{\mu} \\
& =D_{q}^{m} \sum_{k=0}^{\infty} \frac{\left(q^{-\gamma} ; q\right)_{k}}{(q ; q)_{k}}\left(\frac{q^{\alpha}}{z}\right)^{k} \xi^{\mu+k} \\
& =\sum_{k=0}^{\infty} \frac{\left(q^{-\gamma} ; q\right)_{k}}{(q ; q)_{k}}\left(\frac{q^{\alpha}}{z}\right)^{k} \frac{\Gamma_{q}(\mu+k+1)}{\Gamma_{q}(\mu+k-m+1)} \xi^{\mu+k-m} .
\end{aligned}
$$

Hence,

$$
\begin{aligned}
& \left.D_{q}^{m}\left(q^{\alpha-\gamma} \frac{\xi}{z} ; q\right)_{\gamma} \xi^{\mu}\right|_{\xi=z} \\
& \quad=z^{\mu-m} \frac{\Gamma_{q}(\mu+1)}{\Gamma_{q}(\mu-m+1)}{ }_{2} \phi_{1}\left(q^{-\gamma}, q^{\mu+1} ; q^{\mu-m+1} ; q, q^{\alpha}\right) \\
& \quad=(-1)^{m} \frac{\left(q^{-\mu} ; q\right)_{m}}{(1-q)^{m}} q^{m \mu-\left(\begin{array}{c}
m \\
2
\end{array}\right)} z^{\mu-m}{ }_{2} \phi_{1}\left(q^{-\gamma}, q^{\mu+1} ; q^{\mu-m+1} ; q, q^{\alpha}\right)
\end{aligned}
$$

and

$$
\begin{aligned}
\left(I_{q}^{\alpha-\gamma+m} V\right)\left(z q^{m}\right) & =\frac{\Gamma_{q}(\lambda)}{\Gamma_{q}(\lambda+\alpha-\gamma+m)}\left(z q^{m}\right)^{\lambda+\alpha-\gamma+m-1} \\
& =\frac{\Gamma_{q}(\lambda)}{\Gamma_{q}(\lambda+\alpha-\gamma)} \frac{(1-q)^{m}}{\left(q^{\lambda+\alpha-\gamma} ; q\right)_{m}}\left(z q^{m}\right)^{\lambda+\alpha-\gamma+m-1}
\end{aligned}
$$

Then applying Theorem 3.1 gives

$$
\begin{aligned}
I_{q}^{\alpha}(U V)(z)= & z^{\mu+\lambda+\alpha-1} \frac{\Gamma_{q}(\alpha) \Gamma_{q}(\lambda)}{\Gamma_{q}(\alpha-\gamma) \Gamma_{q}(\lambda+\alpha-\gamma)} \\
& \times \sum_{m=0}^{\infty} q^{m(\lambda+\mu)} \frac{\left(q^{\alpha-\gamma}, q^{-\mu} ; q\right)_{m}}{\left(q, q^{\lambda+\alpha-\gamma} ; q\right)_{m}}{ }_{2} \phi_{1}\left(q^{-\gamma}, q^{\mu+1} ; q^{\mu-m+1} ; q, q^{\alpha}\right) .
\end{aligned}
$$

On the other hand,

$$
I_{q}^{\alpha} z^{\lambda+\mu-1}=\frac{\Gamma_{q}(\lambda+\mu)}{\Gamma_{q}(\lambda+\mu+\alpha)} z^{\lambda+\mu+\alpha-1} .
$$

Equating (54) and (55) gives (51). 
Example 4.9 For complex numbers $a, b, A, B, d$, and $D$ such that $\operatorname{Re}(b)>-1, \operatorname{Re}(B)>0$, and $\operatorname{Re}(b+B)>1$,

$$
\begin{aligned}
& { }_{2} \phi_{1}\left(q^{a+A}, q^{b+B} ; q^{d+D} ; q, q^{-(a+A)} z\right) \\
& =(z ; q)_{-a} \frac{\Gamma_{q}(d+D) \Gamma_{q}(B)}{\Gamma_{q}(b+B) \Gamma_{q}(d+B-b)}(1-q)^{B-D} \\
& \quad \times \sum_{m=0}^{\infty}(-1)^{m} q^{\left(\begin{array}{c}
m \\
2
\end{array}\right)} q^{m B} \frac{\left(q^{b-d} ; q\right)_{m}}{(q ; q)_{m}\left(q^{d+D-b} ; q\right)_{m}} \\
& \quad \times{ }_{3} \phi_{2}\left(q^{-m}, q^{d+D-b-B}, z q^{-a} ; q^{d-b}, z ; q, q^{b+1}\right){ }_{2} \phi_{1}\left(q^{A}, q^{B} ; q^{d+B-b+m} ; q, q^{-a-A+m}\right)
\end{aligned}
$$

for $\left|z q^{-a-A}\right|<1$.

Proof The previous identity follows by taking

$$
U(z)=z^{b}(z ; q)_{-a}, \quad V(z)=z^{B-1}\left(z q^{-a} ; q\right)_{-A}
$$

and applying Theorem 3.1 with

$$
\alpha=d+D-b-B, \quad \gamma=D-B .
$$

Then using (3), we obtain

$$
\begin{aligned}
\left.D_{q}^{m}\left(\left(q^{d-b} \frac{\xi}{z} ; q\right)\right)_{D-B} \xi^{b}(\xi ; q)_{-a}\right)\left.\right|_{\xi=z}= & (1-q)^{-m} z^{-m+b}(z ; q)_{-a} \frac{\left(q^{d-b} ; q\right)_{\infty}}{\left(q^{d-b+D-B} ; q\right)_{\infty}} \\
& \times{ }_{3} \phi_{2}\left(q^{-m}, q^{d-b+D-B}, z q^{-a} ; q^{d-b}, z ; q, q^{b+1}\right) .
\end{aligned}
$$

In addition,

$$
\begin{aligned}
\left(I_{q}^{\alpha-\gamma+m} V\right)\left(z q^{m}\right)= & z^{d-b+\beta-1+m} q^{m^{2}+(d-b+\beta-1) m} \frac{\Gamma_{q}(\beta)}{\Gamma_{q}(B+m+d-b)} \\
& \times{ }_{2} \phi_{1}\left(q^{A}, q^{\beta} ; q^{m-b} ; q, q^{m-a-A}\right)
\end{aligned}
$$

and

$$
\left[\begin{array}{c}
\gamma-\alpha \\
m
\end{array}\right]_{q}=(-1)^{m} q^{(\gamma-\alpha) m} q^{\left(\begin{array}{c}
-m \\
2
\end{array}\right)} \frac{\left(q^{\alpha-\gamma} ; q\right)_{m}}{(q ; q)_{m}} .
$$

Substituting with (57)-(59) into (21), we obtain

$$
\begin{aligned}
I_{q}^{\alpha} U V(z)= & z^{d+D-1}(1-q)^{D-B}(z ; q)_{-a} \frac{\Gamma_{q}(\beta)}{\Gamma_{q}(B+d-b)} \\
& \times \sum_{m=0}^{\infty}(-1)^{m} q^{\left(\begin{array}{c}
m \\
2
\end{array}\right)} q^{m B} \frac{\left(q^{b-d} ; q\right)_{m}}{(q ; q)_{m}\left(q^{d+D-b} ; q\right)_{m}} \\
& \times{ }_{3} \phi_{2}\left(q^{-m}, q^{d+D-b-B}, z q^{-a} ; q^{d-b}, z ; q, q^{b+1}\right) \\
& \times{ }_{2} \phi_{1}\left(q^{A}, q^{B} ; q^{d+B-b+m} ; q, q^{-a-A+m}\right) .
\end{aligned}
$$


On the other hand,

$$
I_{q}^{\alpha} U V(z)=\frac{\Gamma_{q}(b+B)}{\Gamma_{q}(d+D)} z_{2}^{d+D-1} \phi_{1}\left(q^{a+A}, q^{b+B} ; q^{d+D} ; q, q^{-(a+A)}\right) .
$$

\section{Combining (60) and (61), we obtain (56).}

\section{Competing interests}

The author declares that they have no competing interests.

\section{Acknowledgements}

This research is supported by NPST Program of King Saud University; project number 10-MAT1293-02.

Received: 5 September 2012 Accepted: 24 January 2013 Published: 11 February 2013

\section{References}

1. Gasper, G, Rahman, M: Basic Hypergeometric Series, 2nd edn. Cambridge University Press, Cambridge (2004)

2. Ismail, MEH: Classical and Quantum Orthogonal Polynomials in One Variable. Cambridge University Press, Cambridge (2005)

3. Jackson, FH: On q-definite integrals. Q. J. Pure Appl. Math. 41, 193-203 (1910)

4. Hahn, W: Beiträge zur Theorie der Heineschen Reihen. Math. Nachr. 2, 340-379 (1949) (German)

5. Al-Salam, WA: Some fractional $q$-integrals and $q$-derivatives. Proc. Edinb. Math. Soc. 2(15), 135-140 (1966/1967)

6. Al-Salam, WA: q-analogues of Cauchy's formulas. Proc. Am. Math. Soc. 17, 616-621 (1966)

7. Agarwal, RP: Fractional q-derivative and $q$-integrals and certain hypergeometric transformations. Ganita 27(1-2), 25-32 (1976)

8. Purohit, SD: On a q-extension of the Leibniz rule via Weyl type of q-derivative operator. Kyungpook Math. J. 50(4), 473-482 (2010)

9. Agarwal, RP: Certain fractional $q$-integrals and q-derivatives. Proc. Camb. Philos. Soc. 66, 365-370 (1969)

10. Annaby, MH, Mansour, ZS: 9 -Fractional Calculus and Equations. Lecture Notes of Mathematics, vol. 2056. Springer, Berlin (2012). doi:10.1007/978-3-642-30898-7

11. Abdeljawad, T, Baleanu, D: Caputo $q$-fractional initial value problems and a $q$-analogue of Mittag-Leffler function. Commun. Nonlinear Sci. Numer. Simul. 16(12), 4682-4688 (2011)

12. Liouville, J: Mèmoire sur le calcul des différentielles à indices quelconques. J. Éc. Polytech. 13, 71-162 (1832)

13. Grünwald, AK: Über begrenzte derivationen und deren anwedung. Z. Angew. Math. Phys. 12, 441-480 (1867)

14. Letinkov, AV: Theory of differentiation of fractional order. Mat. Sb. 3, 1-68 (1868)

15. Watanabe, Y: Notes on the generalized derivative of Riemann-Liouville and its application to Leibniz's formula. I and II. Tohoku Math. J. 34, 8-41 (1931)

16. Post, EL: Generalized differentiation. Trans. Am. Math. Soc. 32, 723-781 (1930)

17. Bassam, MA: Some properties of Holmgren-Riez transform. Ann. Sc. Norm. Super. Pisa 15(3), 1-24 (1961)

18. Gaer, MC, Rubel, LA: The fractional derivative and entire functions. In: Fractional Calculus and Its Applications (Proc. Internat. Conf., Univ. New Haven, West Haven, Conn., 1974). Lecture Notes in Math., vol. 457, pp. 171-206. Springer, Berlin (1975)

19. Osler, TJ: Leibniz rule for fractional derivatives generalized and an application to infinite series. SIAM J. Appl. Math. 18(3), 658-674 (1970)

20. Osler, TJ: Fractional derivatives and Leibniz rule. Am. Math. Mon. 78(6), 645-649 (1971)

21. Diaz, JB, Osler, TJ: Differences of fractional order. Math. Comput. 28(125), 185-202 (1974)

22. Osler, TJ: The integral analogue of the Leibniz rule. Math. Comput. 26(120), 903-915 (1972)

23. Osler, TJ: A correction to Leibniz rule for fractional derivatives. SIAM J. Math. Anal. 4, 456-459 (1973)

24. Herrmann, R: Fractional Calculus: An Introduction for Physicists. World Scientific, Singapore (2011)

25. Baleanu, D, Diethelm, K, Scalas, E, Trujillo, Jj: Fractional Calculus Models and Numerical Methods. Series on Complexity, Nonlinearity, and Chaos, vol. 3. World Scientific, Singapore (2012)

26. Baleanu, D, António, J, Machado, T, Luo, ACJ: Fractional Dynamics and Control. Springer, Berlin (2012)

27. Golmankhaneh, AK: Investigations in Dynamics: With Focus on Fractional Dynamics. LAP Lambert Academic Publishing, Saarbrücken (2012)

28. Hilfer, R (ed.): Applications of Fractional Calculus in Physics. World Scientific, Singapore (2000)

29. Al-Salam, WA, Verma, A: A fractional Leibniz q-formula. Pac. J. Math. 60(2), 1-9 (1975)

30. Ramis, JP: About the growth of entire functions solutions of linear algebraic $q$-difference equations. Ann. Fac. Sci. Toulouse 1(6), 53-94 (1992)

31. Purohit, SD: Summation formulae for basic hypergeometric functions via fractional $q$-calculus. Matematiche 64(1), 67-75 (2009)

32. Manocha, HL, Sharma, BL: Fractional derivatives and summation. J. Indian Math. Soc. 38(1-4), 371-382 (1974)

33. Lighthill, MJ: Introduction to Fourier Analysis and Generalized Functions. Cambridge University Press, New York (1960)

34. Miller, KS, Ross, B: An Introduction to the Fractional Calculus and Fractional Differential Equations. Wiley, New York (1993) 